\title{
Fabrication of nanoporous junctions using off-the-shelf Nafion membrane
}

\author{
Dinh-Tuan Phan, ${ }^{\mathrm{a}}$ Chun Yang, ${ }^{\text {a }}$ and Nam-Trung Nguyen ${ }^{\text {*b }}$
}

${ }^{a}$ School of Mechanical and Aerospace Engineering, Nanyang Technological University, 50 Nanyang Avenue, Singapore 639798. Email: mcyang@ntu.edu.sg

${ }^{b}$ Queensland Micro- and Nanotechnology Centre, Griffith University, Brisbane, 4111, Australia. E-mail: nam-trung.nguyen@griffith.edu.au

\section{Abstract}

This paper reports a method to fabricate nanoporous junctions in microfluidic devices using off-the-shelf Nafion membrane. These nanoporous junctions are needed for various applications based on the phenomenon of ion concentration polarisation (ICP). The advantages of this unique method compared to other fabrication methods of cation selective nanofluidic junctions are the simpler fabrication process, the better durability, and the increase in ICP repulsion force. Thus, microfluidic devices fabricated with that method have the potential to enhance the separation efficiency of the ICP process. 


\section{Introduction}

Ion concentration polarisation (ICP) is a fundamental electrochemical transport phenomenon that occurs when ion current passes through an ion-selective membrane. Often called ion depletion or enrichment process [1], this phenomenon happens due to the mismatch of charged carriers at the membrane interface. The membrane (either nanochannel or nanoporous membrane) preferentially or only conducts one sign of ion such as cations in cation exchange membrane. As a result, ion concentration gradients are generated on both sides of the membrane. Once ICP is triggered near the cation exchange membrane interface, the concentrations of both cations and anions decrease on the anodic side of the junction (i.e. ion depletion) and increase on the cathode side (i.e. ion enrichment). Furthermore, any charged particles, cells and other small colloids will be similarly depleted or enriched in the same analogy.

Recently, ICP has attracted a great deal of attention from the lab-on-a-chip research community because of its potential applications such as sample concentration [2-5], desalination $[6,7]$, separation and mixing $[8,9]$. Further applications of ICP have been reported recently. Jeon et al [10] presented a novel separation method, which can continuously separate the particles based on the ICP phenomenon and the electrophoretic mobility of micro- and nano-sized particles. MacDonald et al [7] reported a method of out-of-plane ion concentration polarization for scalable water desalination, which has the advantages of increasing the throughput and the ability of integrating multiple devices in a single system. Most of the previously reported ICPbased devices used Nafion resin to deposit a thin nanoporous membrane with a thickness varying from $191 \mathrm{~nm}$ using microflow patterning method [2] to $200 \mu \mathrm{m}$ by filling a laser-cut micro channel [7].

The advantage of the fabrication method of depositing a cation-selective junction inside microfluidic devices using Nafion resin as reported by Han's group [2-6] is the quick formation of a thin Nafion layer on a clean glass slide. However, this method requires an extra PDMS microchannel serving as a master mould and needs several fabrication steps. We observed that this deposition process was not straightforward as 
expected. The limitations are as follow. The negative pressure required for sucking out the Nafion solution needs to be controlled accurately. The Nafion film does not adhere firmly on the glass slide and can peel off easily. The edges and surface of the fabricated Nafion trip are not smooth. Moreover, the thickness and width of the deposited Nafion trip could not be controlled precisely since the evaporation unpredictably of solution under the heating process.

To simplify the fabrication process, we used an off-the-shelf Nafion membrane (NR212, DuPont Co., USA) with a thickness of $50 \mu \mathrm{m}$ instead of the Nafion resin to deposit a thin cation-selective membrane on a glass substrate. This off-the-shelf membrane provides better mechanical durability and a longer working time. Moreover, a thicker membrane generates a stronger electrical repulsive force around the membrane [4]. In this paper, we report a unique and simple fabrication method to integrate a thin off-theshelf Nafion strip into a microfluidic device for applications with ICP. We chose the fabrication of a separation device to demonstrate and compare the fabrication process and the performance of this new method with the published work. The separation principle is based on the desalination concept introduced previously by Kim et al [6] and depicted in Figure 1(a). The desalination process using ICP to split seawater into a desalted stream and a concentrated stream by passing the seawater over, not through, an ion-selective membrane. The ion depletion zone above the membrane can block off ions and other charged species, but allowing desalted water to go through. In this device, the most important component is the cation-selective membrane. We aim to fabricate these devices using off-the-shelf Nafion membrane to overcome the current drawbacks of the Nafion resin. 


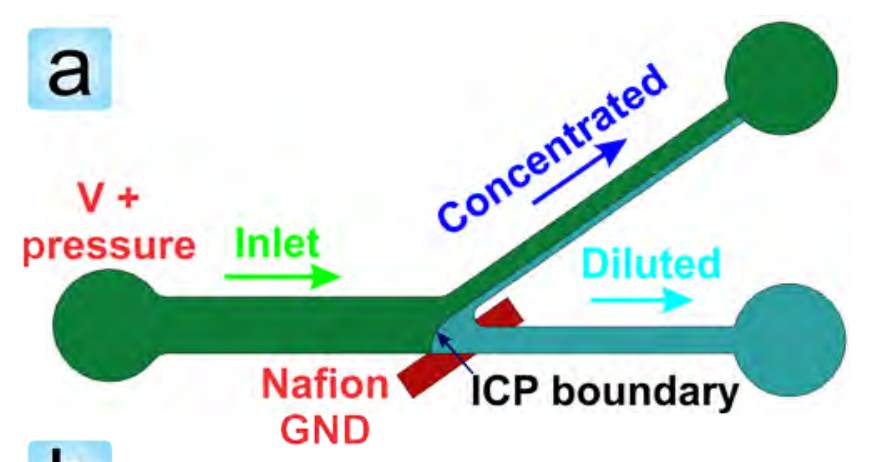

b

(i) Glass

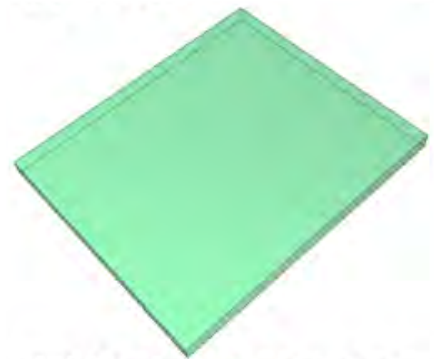

(iii) Nafion membrane

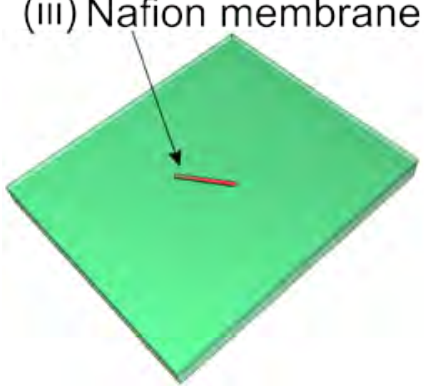

(ii) PDMS spin coating

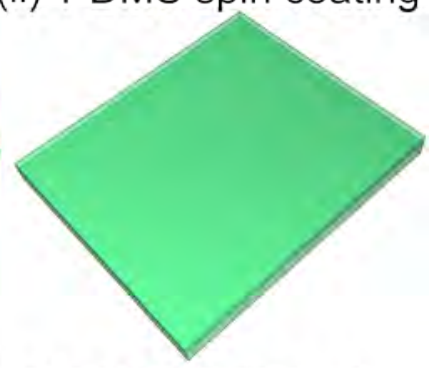

(iv) PDMS channel

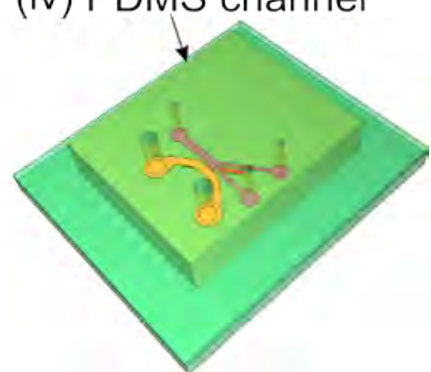

Figure 1. The separation device: (a) Working concept of the device. (b) Integration of a 50- $\mu \mathrm{m}$ thick Nafion membrane on a PDMS/glass substrate. (i) Preparing and cleaning a glass slide. (ii) Coating the PDMS mixture with a spin speed of $1450 \mathrm{rpm}$. (iii) Cutting the Nafion membrane and placing it carefully on top of the PDMS surface. Placing the sample at room temperature overnight to let PDMS reflow to form a flat surface. Next, placing the sample into the oven at $80^{\circ} \mathrm{C}$ for 2 hours. (iv) Bonding the top PDMS with microchannel network to the PDMS/glass substrate with the Nafion membrane using a precision alignment system.

\section{Methods}

We fabricated the microfluidic device for demonstration of a separation process with an integrated cation selective membrane using two different methods, to compare the two fabrication concepts. To create a nanoporous junction, the previously reported 
desalination device used a Nafion resin to fill a microchannel [6] (thereafter called as resin-made-device). Our proposed method uses an off-the-shelf Nafion strip integrated onto a thin polydimethylsiloxane (PDMS) layer (thereafter called as membrane-madedevice).

\subsection{Nanojunction based on Nafion resin}

Figures 1(a) and (b) show the configuration of the separation device and the layers of the device fabricated by the proposed method, respectively. With this configuration, we first fabricated the devices using the Nafion resin. The fabrication process is summarized as follows. A square glass slide served as the substrate for the sevice. Gold electrodes with a thickness of $300 \mathrm{~nm}$ were sputtered on the glass substrate. The thickness of deposited electrode is a compromise between reducing the electrical resistance and minimizing the gap between the PDMS channel layer and glass substrate for leakage-free bonding. The sputtered electrodes consist of two layers. A $50 \mathrm{~nm}$-thick titanium layer was used to enhance the adhesion of the gold layer to the glass substrate. A $250 \mathrm{~nm}$-thick gold layer was deposited on top of this adhesion layer. Gold was selected due to its good conductivity and its resistance to oxidation. The Nafion junction was formed by the microflow patterning technique [2]. This technique used a PDMS mould having a microchannel with a width of $100 \mu \mathrm{m}$ and a height of $10 \mu \mathrm{m}$. The mould was temporarily bonded on the glass substrate. The microchannel was then filled with $10 \mathrm{wt} \%$ solution of Nafion perfluorinated ion-exchange resin (Sigma Aldrich, St. Louis, USA). Negative pressure was applied on the microchannel outlet first to fill it and then to suck out the resin to control the deposited thickness. Subsequently, the sample was baked in an oven at $110{ }^{\circ} \mathrm{C}$ for 10 minutes to cure the deposited Nafion strip. The top layer of the device was made of PDMS and contains the microfluidic channel network fabricated by the standard soft lithography process. Negative photoresist SU-8 (MicroChem Corp., USA) with a thickness of $100 \mu \mathrm{m}$ was first coated on a silicon wafer. The channel features were defined by exposing the SU-8 layer through a mask to UV light. PDMS channel was casted on, cured and then peeled off from the SU-8 mould. Finally, the surfaces of the PDMS part and the glass substrate were treated with 
oxygen plasma (Harrick PDC-32G) for one minute before bonding together with the help of the precise alignment system described in the later section.

\subsection{Nanojunction based on off-the-shelf Nafion membrane}

To compare the efficiency of separation process in the devices fabricated with the two different methods, we used the same channel dimension to fabricate another device with our proposed method, as described in details below. Sylgard 184 and a curing agent (Dow Corning Inc.) were mixed in a 10:1 ratio by weight. The mixture was degassed for one hour in a vacuum chamber. Subsequently, $5 \mathrm{ml}$ of the PDMS mixture was poured onto a square glass slide and spin coated in two steps: $500 \mathrm{rpm}$ for 10 seconds to spread the PDMS over the surface, and then increase to $1450 \mathrm{rpm}$ for 30 seconds. Figure 2 shows the measured height of the Nafion membrane relative to the PDMS surface. The results show that the mismatch between the PDMS surface and the Nafion surface is proportional to the spin speed. Tuning the spin speed can match the surface of the Nafion and the surrounding PDMS.
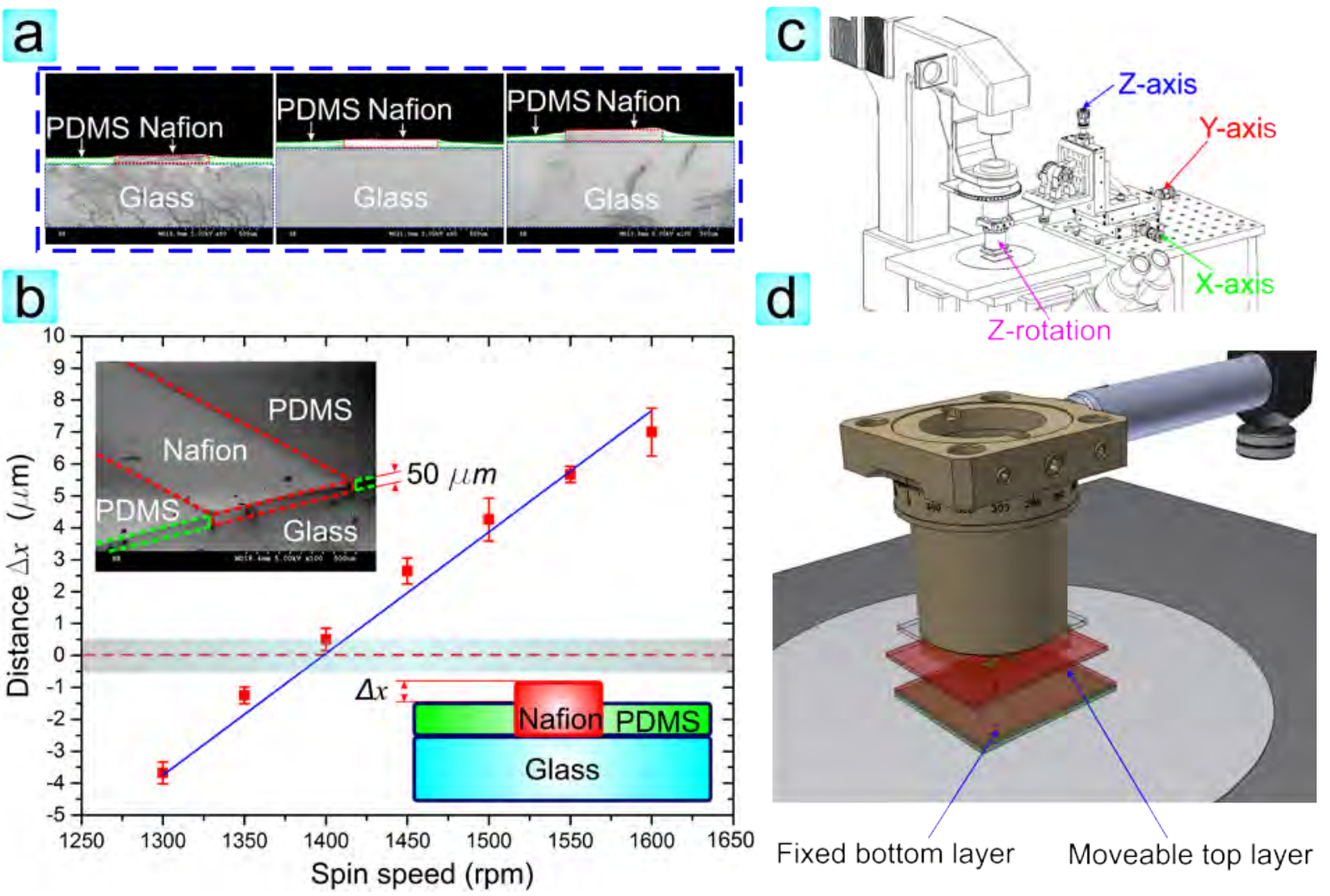
Figure 2. Fabrication of Nafion membrane-made-device: (a) SEM images are the cross section views of a glass slide coated with PDMS after integrating with a Nafion strip. The images from left to right indicate different spin speeds for PDMS coating, 1400, 1450 and $1500 \mathrm{rpm}$, respectively. (b) The relative height between PDMS surface and Nafion surface coated on a glass substrate by varying different spin speeds. The fitting line (solid) shows the linear relationship between the spin speed and the distance $\Delta x$. Insets are SEM images showing the isometric view (top left corner) and the defined distance $(\Delta x)$ between PDMS surface and Nafion surface (bottom right corner). The dashed line shows the reference at which with $\Delta x=0$. The grey band is considered experimentally as the leakage-free region for bonding between the PDMS channel and the coated substrate. (c) Alignment system for bonding of the fabricated devices mounted next to the microscope. The whole system has an estimated alignment error below $1 \mu \mathrm{m}$. (d) Closer view of the alignment equipment with four degrees of freedom. Three transition movements $\mathrm{X}, \mathrm{Y}, \mathrm{Z}$ and one rotation movement around $\mathrm{Z}$ axis were used to precisely position the top PDMS part to the bottom glass substrate.

Next, a thin Nafion trip was placed on top of this spin-coated surface and kept in a clean place overnight to allow PDMS to reflow and form a flat surface. To make sure that the thin PDMS film is evenly coated, the substrate can be spin coated again before curing in an oven. The microchannels of the device were fabricated following a general PDMS soft-lithography process. The master mould was made with SU-8 photoresist (MicroChem Corp., USA) and standard photolithography process. After peeling off from the mould, the PDMS part was bonded to the PDMS-glass substrate with the integrated a Nafion membrane using oxygen plasma treatment and a precise alignment system, Figure 2(c, d). An aligner was designed and assembled from the Thorlab, Inc. (USA) standard components, to position of the Nafion strip accurately against the concentrated channel, Figure 1(a). The alignment process was performed as follows. First, both PDMS top and bottom layers were cleaned by oxygen plasma for 45 seconds. Then, the PDMS-glass substrate integrated with Nafion strip was quickly placed on the sample holder of the microscope. Concurrently, the top PDMS microchannel layer was attached on the sample holder of the alignment head, Figure 2(d). Next, adjusting the four degrees of freedom (three translations and one rotation) brought the Nafion trip to the right position parallel to the concentrated channel. During the alignment process, we could observe the structures to be aligned either via microscope eyepieces or a camera attached to the microscope. From the experimental results, the alignment gap could be 
achieved in the range from $5 \mathrm{zu} 10 \mu \mathrm{m}$, Figure 3(c). Since the active PDMS surface would disappear over time, all steps need to be performed quickly. With our alignment system, the whole process only took 1015 seconds.
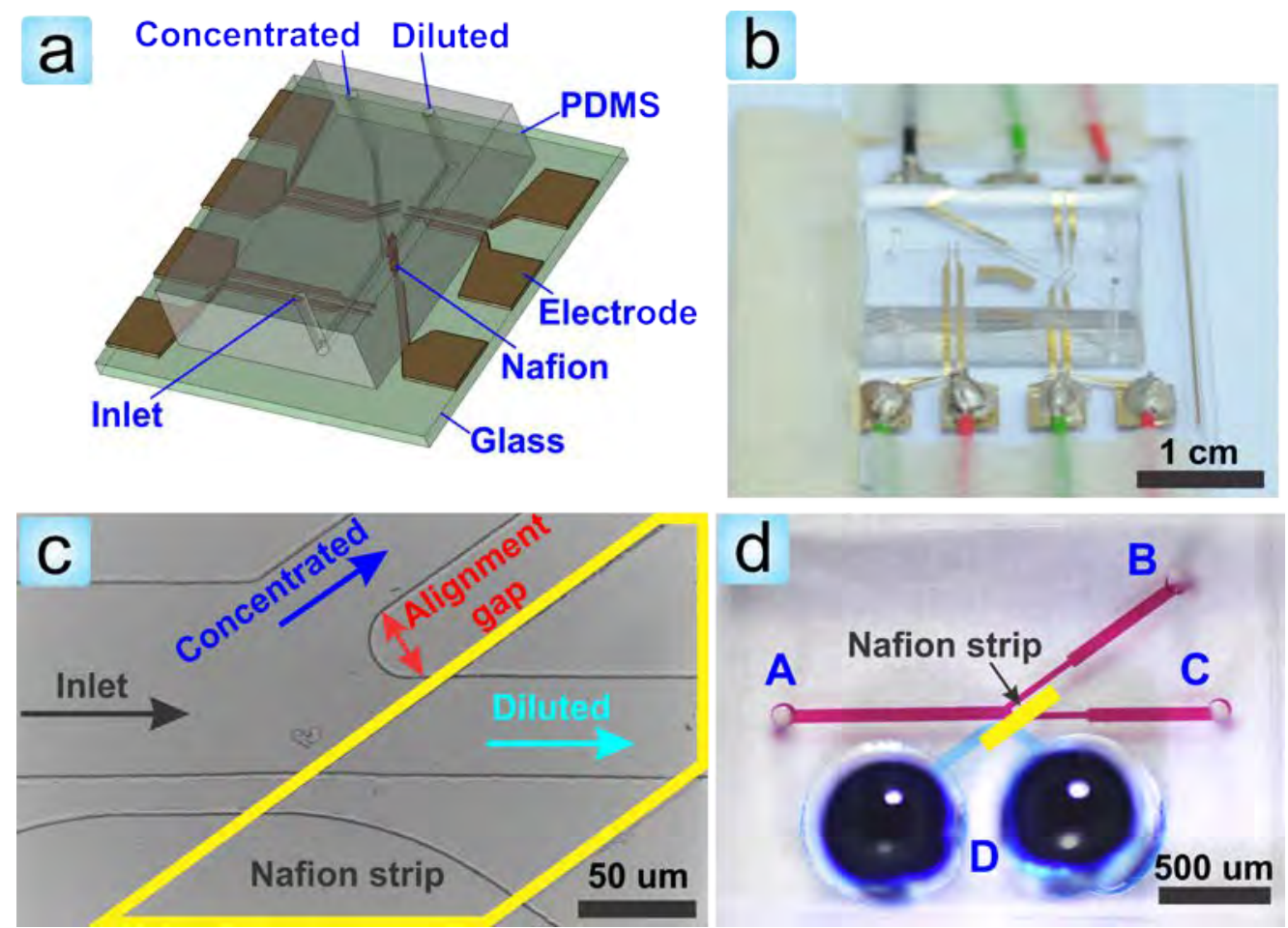

Figure 3. Fabrication of devices. (a) Design of the microfluidic separation device used Nafion resin to deposit a thin cation selective nanojunction. (b) The fabricated device shows electrical connections to the gold pads sputtered on a glass substrate. (c) The separation configuration was used to test the new fabrication method, in which off-the-shelf Nafion membrane was integrated into a thin PDMS layer coated on a glass substrate. The top PDMS microchannel was kept unchanged. The microscopy image shows the alignment of Nafion membrane (slanted strip bounded in the yellow lines) parallel to the concentrated channel under a precise alignment system. This step is performed during the plasma bonding process. The alignment gap indicates the allowed error defined by the distance between the outermost edge of the Nafion membrane and the opposite channel edge. (d) Fabricated microfluidic device using off-the-shelf Nafion membrane filled with food dyes.

Figures 3(a) and (b) depict the device design and the fabricated device, respectively. Figure 3(c) shows the position of the Nafion strip relative to other microchannels, which are visualised by food dye in Figure 3(d). The Nafion strip (yellow) was integrated with 
a thin PDMS layer and aligned parallel to the concentrated channel. Red channels were inlet (A) connected to $\mathrm{V}+$, concentrated outlet (B) and diluted outlet (C). The blue channel was the buffer solution PBS connected to GND.

\subsection{Experimental set up}

The device was subsequently tested for its functionality. In our experiments, all liquids were kept in 5-ml Hamilton glass syringes, which were driven by syringe pumps (neMESYS, Cetoni, Germany) to provide the pre-set flow rates to the device. A highspeed CMOS camera (Phantom Miro eX4) attached to an inverted epi-fluorescent microscope (Nikon Eclipse TE2000-S) was used to capture the fluorescent images of the channels. The colour images were taken by a DSLR camera (Nikon D700) mounted on the right camera port of the microscope via a specialized adapter combining with an extension tube. A solution of $10 \mu \mathrm{M}$ fluorescein sodium salt $\mathrm{C}_{20} \mathrm{H}_{10} \mathrm{Na}_{2} \mathrm{O}_{5}$ (Sigma Aldrich, St. Louis, USA) in DI water (MiliQ system, Milipore, USA) was used as the inlet sample. We also prepared another sample with a concentration of $500 \mu \mathrm{M}$ to measure the reference fluorescence intensity value. For the experiments on devices used the off-the-shelf Nafion membrane, the phosphate buffer solution (PBS) with the concentration of $100 \mu \mathrm{M}$ was used to fill the buffer channel for the electric ground. The prepared solution had a $\mathrm{pH}$ value of approximately 8 and a conductivity of $0.75 \mathrm{mS} \mathrm{cm}^{-1}$ measured by a portable $\mathrm{pH} /$ conductivity meter kit (Thermo Scientific Orion 4-Star Plus, USA). Since most ions are rejected from the diluted outlet and collected at the concentrated outlet, the $\mathrm{pH}$ value of the concentrated stream was much higher than that of the diluted stream and the sample flow. For calibration purpose, we also measured the conductivities at various concentrations. The fluorescein sodium salt solutions with concentrations of 100 and $500 \mu \mathrm{M}$ have corresponding conductivities of 1.9 and $7.5 \mathrm{mS}$

$\mathrm{cm}^{-1}$, respectively. The electric fields were provided by a high voltage DC power supply (Model PS350, Stanford Research System, Inc.). The fluorescent dye has an excitation wavelength of $540 \mathrm{~nm}$ and an emission wavelength of $610 \mathrm{~nm}$. The separation process was observed with the help of a filters set consisted an excitation filter for $540 \mathrm{~nm}$, a dichroic mirror for $565 \mathrm{~nm}$ and an emission filter for $605 \mathrm{~nm}$. After recording the 
images with the high-speed camera, the fluorescence intensity and distance were analysed by using a customized MATLAB program.

\section{Results and discussions}

\subsection{Separation process}

We characterised the fluorescence intensity at the area located in front of the Nafion junction to demonstrate the continuous separation. Once the ICP is triggered, all charged ions are repelled away from the Nafion membrane and formed a boundary to prevent any charged species to enter this region. In order to compare the separation performance of the two fabricated devices, the fluorescence intensities have been normalized to the intensity of the $10-\mu \mathrm{M}$ input sample used in the device with off-the-shelf membrane. Figure 4 shows the normalized fluorescent signals at representative locations close to the junction in both devices. Three lines A, B, C were defined at the input stream, preconcentrated plug, and concentrated stream, respectively. The device made of the off-the-shelf membrane demonstrated a significant enhancement in separation efficiency. Figure 4(b) indicates that the device with off-the-shelf membrane had a 50fold concentration enhancement, which was much higher than the resin-made-device, Figure 4(a). Comparing the preconcentrated curves (B) of these two devices shows that the membrane-based device provides a sharper localized boundary at the ICP region, whereas the surrounding charges adjacent to the ICP boundary in resin-based device was not localized. The charged species were dispersed thus the starting part of the fluorescence intensity curve $B$ in resin-based device was brighter than that of the membrane-based device. This might be a result of the stronger repulsion force caused by a higher conductance in the nanojunction fabricated from the Nafion membrane. The material purity and the uniform pore size of the fabricated junction are believed to be the main contribution factors. Moreover, we noticed that the lower intensity in the later part of the preconcentrated curve (B) proved a higher improvement in charged species rejection of the device based on the Nafion membrane. This behaviour may be useful for the separation processes utilizing ICP. Importantly, the separation efficiency would be improved if optimizing the channel geometries, especially at the bifurcated branches. 
For instance, the concentrated stream (i.e. slanted channel) can be narrowed down to enhance the concentration factor contributed by the geometrical factor, as reported recently on the concentrators utilizing ICP $[5,11]$.
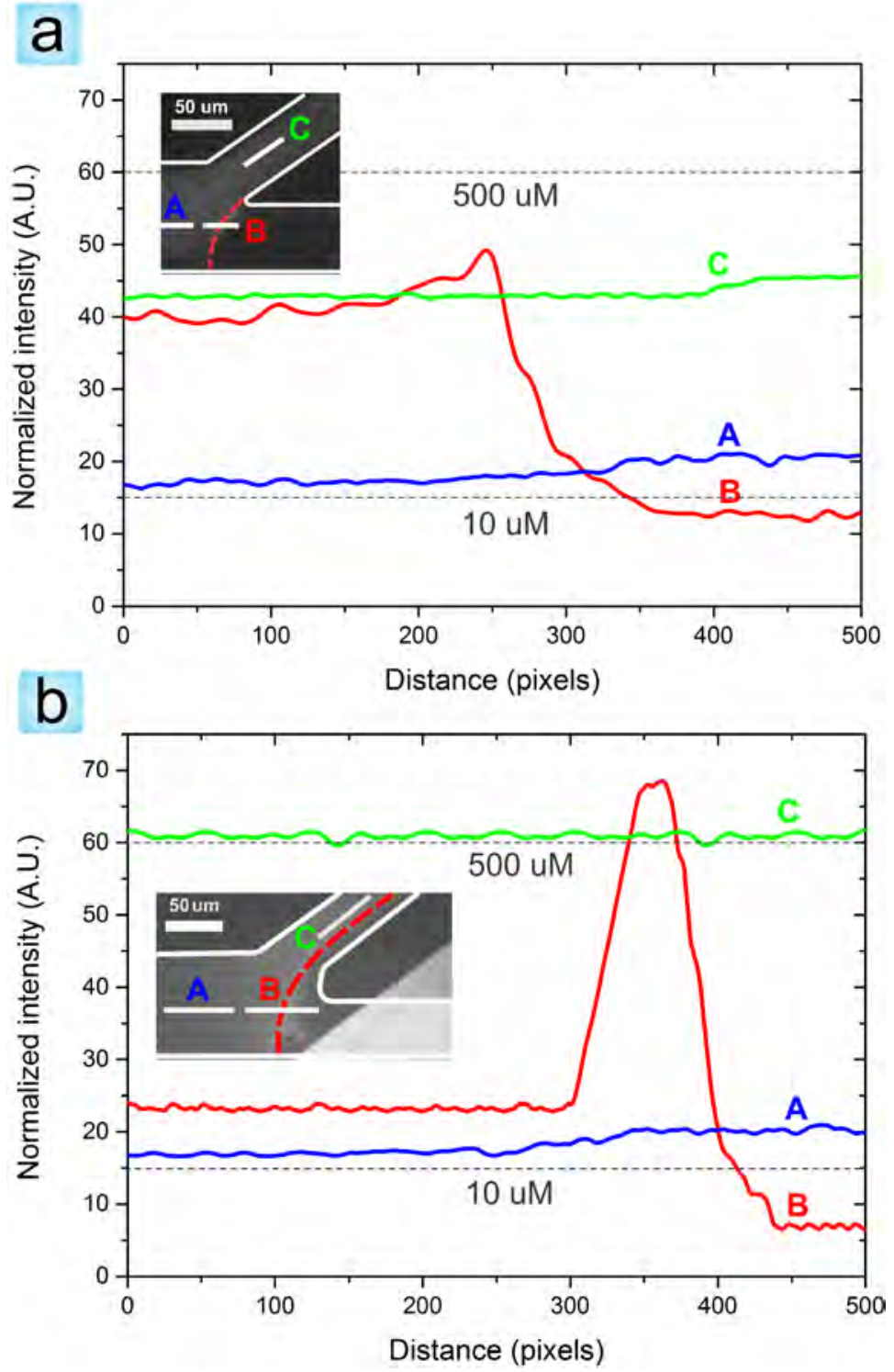

Figure 4. Normalized fluorescence intensity curves in the separation devices fabricated by (a) Nafion resin and by (b) off-the-shelf Nafion membrane. All curves are smoothed by adjacent averaging method of 25 sampling points for noise reduction. The dash lines are the reference intensities for calibration concentrations at $10 \mu \mathrm{M}$ and $500 \mu \mathrm{M}$. The normalized fluorescence intensity curves show a jump in the concentration of fluorescein sodium salt at the preconcentrated zone. Generations of the concentrated flow (i.e. the green line C), the concentrated plug (i.e. the peak of the red line B), and the sample flow (i.e. the blue line A) are well-defined. The positions of these three lines are located as shown in the inset images (not 
drawn to scale). The diluted flow profile can be considered as the later part of the intensity curve $\mathrm{B}$, which has a lower concentration than the sample flow.

\subsection{ICP boundary displacement}

Figure 5 shows the measured distance between the ICP boundary and the edge of the Nafion membrane in the Nafion-resin device (circles) and the off-the-shelf Nafion membrane device (squares), respectively. At first, the distance $\Delta x$ is almost linear to the applied voltage. Increase the applied voltage did not shift the ICP boundary further as a result of the limiting current behaviour. Obviously, under the same input conditions, the new fabrication technique provided stronger ICP force and thus repels charges and ions stronger, Figure 5(b).

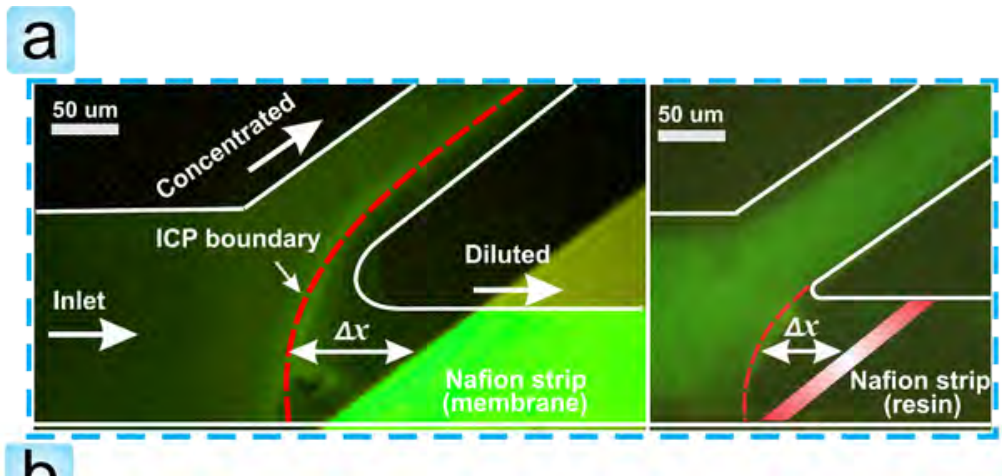

$\mathrm{b}$

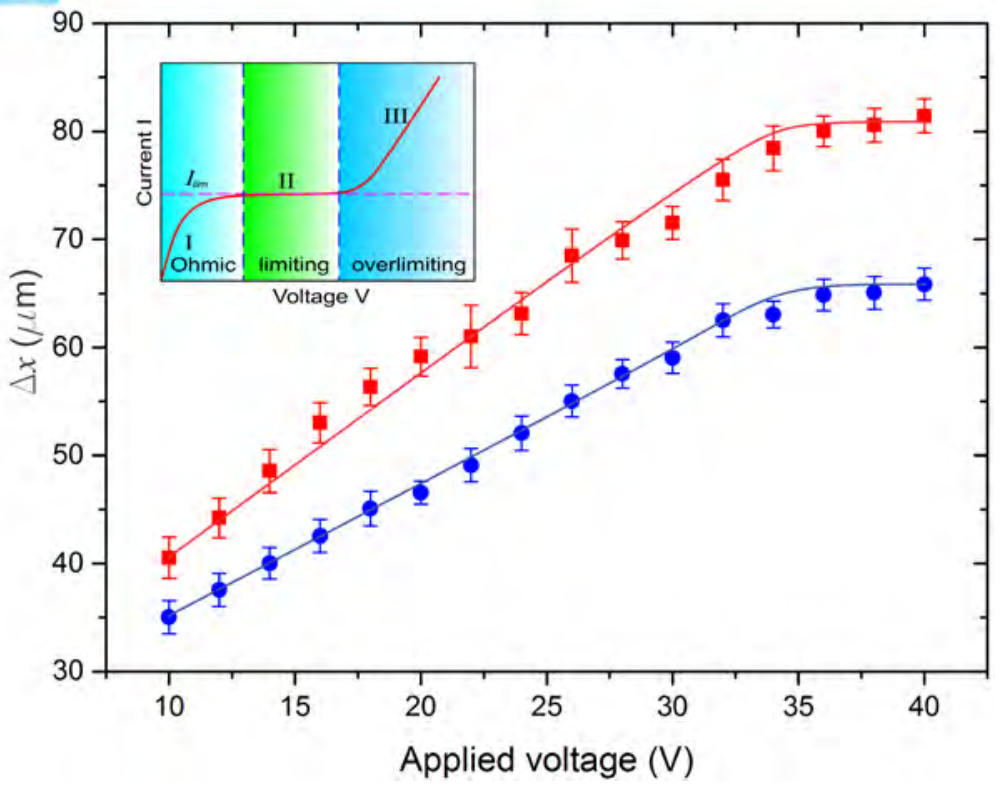


Figure 5. ICP boundary characterization under various applied voltages and fixed input flow rate. (a) The ICP boundary formed under an applied voltage and the generation of the concentrated stream (i.e. flow in the slanted channel) and the diluted stream (i.e. flow in the later section of the horizontal channel). The distance denoted in $\Delta x$ was measured from the Nafion junction edge to the outermost of the ICP boundary. Two separation devices were fabricated from off-the-shelf Nafion membrane (left) and Nafion resin (right). (b) Controlling the position of the ICP boundary by varying different applied voltages. Circles are the results of the device fabricated by Nafion resin. Squares are the results of the device fabricated by off-the-shelf Nafion membrane. The solid lines are the fitting line, which are predicted in the relationship with the typical I-V characterization of ICP phenomenon as shown in the inset graph.

Experimentally, we also observed that the ICP boundary fluctuated significantly and may disappear if the applied voltage is too high. These effects could presumably be explained by the breakdown of the PDMS substrate under the threshold voltage. In this situation, short circuit occurred leading to a significant increase in the current, which was monitored continuously by an ammeter (Keithley). In our experiments, the current was limited to $1 \mu \mathrm{A}$ by the DC power supply. Therefore, the applied voltage can only be varied in a limited range. Importantly, with our proposed method, a clear ICP boundary and nice alignment position of Nafion trip can be observed as shown in the left image in Figure 5(a).

\subsection{Operation time}

We also experimentally tested the two types of devices for continuous operation under the same input parameters. We fixed the flow rate at $100 \mu \mathrm{L} \mathrm{min} \operatorname{mon}^{-1}$ and an applied electric field at $100 \mathrm{~V} \mathrm{~cm}^{-1}$. In this experiment, we intentionally test the devices at high input values to check the lifetime of device. We observed that devices made with Teflon solution could work properly only for 30 minutes, after that the Nafion junction became ineffective. The edges of the Nafion junction were dissolved slowly. ICP boundary became significantly unstable and disappeared after $1-2$ minutes. The malfunction of the junction is probably caused by the lack of structural integrity of the Nafion junction deposited by solution. In contrast, devices made with off-the-shelf Nafion membrane run continuously for more than 45 minutes without any leakages or removal of the 
Nafion strip. It demonstrated the integrity of the self-sealing Nafion strip embedded in PDMS layer. Hypothetically, the operation time is considered as depending strongly on the input flowrate and applied electric field. In our test, the device was operated at higher input values of applied electric field and flowrate $\left(100 \mathrm{~V} \mathrm{~cm}^{-1}\right.$ and $\left.100 \mu \mathrm{L} \mathrm{min}^{-1}\right)$ than the previous work $\left(75 \mathrm{~V} \mathrm{~cm}^{-1}\right.$ and $\left.0.1-20 \mu \mathrm{L} \min ^{-1}\right)$ [6]. To increase the throughput and lengthen the operation time, especially for the real applications requiring the scale-up or multiplexed configuration such as desalination, the wider channels combined with a wider Nafion strip can be applicable. By optimizing the balance between the channel height, Nafion dimensions, sample flowrate, and applied electric field, the ICP boundary could be positioned properly leading to a significant increase in the operation time of the device.

\section{Conclusions}

We have demonstrated a robust method to fabricate the microfluidic devices for the applications of ICP phenomenon using an off-the-shelf Nafion membrane. Two types of separation devices were fabricated using Nafion resin and off-the-shelf Nafion membrane to compare their performance. Ease of fabrication, low cost, and reliability are the advantages of the new fabrication method. The devices can be fabricated thanks to the help of a customised precise alignment system for the bonding process. This system allowed positioning the Nafion trip accurately in place against the microchannel in the top layer. Under the same channel dimension and input parameters, the device offthe-shelf Nafion membrane provided a stronger ICP repulsion force than that fabricated with Nafion resin. Thus, the separation efficiency could be enhanced. Moreover, the use of off-the-shelf Nafion membrane leads to better mechanical integrity and a longer lifetime of the device compared to the existing method. The failure of the junction made with Nafion resin may be caused by the poor adhesion to the glass slide. The off-theshelf Nafion strip provides a better mechanical durability because it is strongly embedded into the PDMS-glass substrate by the self-sealing reflow process of PDMS. Since our device worked under the combination of a hydrodynamic force and an electric field, a Nafion membrane would be more suitable for high flow rates and high applied voltages than the thin Nafion junction formed by resin. Our fabrication method is 
especially useful for continuous concentration [11] or separation process, whereas larger charges and ions need to be filtered out to one outlet. The fabrication method can also be applied to mixing applications required strong electroconvection under ICP zone. The current limitation of this proposed method is the manual placing of Nafion strip on the spin coated PDMS-glass substrate. This issue can be improved by using a customised and automated aligner to scale up the current approach.

\section{Acknowledgements}

D.T.P acknowledges the support from the Nanyang Technological University $\mathrm{PhD}$ Scholarship via Nanyang Environment \& Water Research Institute (NEWRI). N.T.N acknowledges partial funding from Australian Research Council through the grant LP150100153 for the development of the alignment system for bonding of microfluidic devices.

\section{References}

[1] Pu Q, Yun J, Temkin H and Liu S 2004 Ion-Enrichment and Ion-Depletion Effect of Nanochannel Structures Nano Lett. 4 1099-1103

[2] Kim S J, Song Y A and Han J 2010 Nanofluidic concentration devices for biomolecules utilizing ion concentration polarization: Theory, fabrication, and applications Chem. Soc. Rev. 39 912-22

[3] Ko S H, Kim S J, Cheow L F, Li L D, Kang K H and Han J 2011 Massively parallel concentration device for multiplexed immunoassays Lab Chip 11 1351-1358

[4] Ko S H, Song Y A, Kim S J, Kim M, Han J and Kang K H 2012 Nanofluidic preconcentration device in a straight microchannel using ion concentration polarization Lab Chip $124472-82$

[5] Kwak R, Kim S J and Han J 2011 Continuous-flow biomolecule and cell concentrator by ion concentration polarization Anal. Chem. 83 7348-55

[6] Kim S J, Ko S H, Kang K H and Han J 2010 Direct seawater desalination by ion concentration polarization Nat. Nanotechnol. 5 297-301 
[7] MacDonald B D, Gong M M, Zhang P and Sinton D 2014 Out-of-plane ion concentration polarization for scalable water desalination Lab Chip 14 681-685

[8] Jännig O and Nguyen N T 2011 A polymeric high-throughput pressure-driven micromixer using a nanoporous membrane Microfluid. Nanofluid. 10 513-519

[9] Kim D, Raj A, Zhu L, Masel R I and Shannon M A 2008 Non-equilibrium electrokinetic micro/nano fluidic mixer Lab Chip 8 625-628

[10] Jeon H, Lee H, Kang K H and Lim G 2013 Ion concentration polarization-based continuous separation device using electrical repulsion in the depletion region Sci. Rep. 33483

[11] Phan D T, Yang C and Nguyen N T 2015 A continuous-flow droplet-based concentrator using ion concentration polarization RSC Adv. 5 44336-44341 